\section{TATRA \\ MOUNTaiNS \\ Mathematical Publications}

DOI: $10.2478 /$ tmmp-2013-0008

Tatra Mt. Math. Publ. 54 (2013), 93-99

\title{
CONDITIONS FOR FACTORIZATION OF LINEAR DIFFERENTIAL-DIFFERENCE EQUATIONS
}

\author{
Klara R. Janglajew - Kim G. Valeev
}

ABSTRACT. The paper deals with a linear system of differential equations of the form

$$
\frac{d X(t)}{d t}=A X(t)+\mu \sum_{k=1}^{n} A_{k} X\left(t+\tau_{k}\right)
$$

with constant coefficients, a small parameter and complex deviating argument. Sufficient conditions for factorizing of this system are presented. These conditions are obtained by construction of an integral manifold of solutions to the considered system.

\section{Introduction and preliminaries}

Factorization of linear differential and difference operators is an effective method for analyzing linear ordinary differential and difference equations, see for instance [8, [10, [14].

Methods of factorization are dedicated to analytical and algebraic approaches to the problem of the integration of ordinary differential equations (see [2] and the references given there).

The contents of this paper is connected to the study of asymptotic properties of delay differential equations. There is a number of important papers on the subject, and we only mention a few: [3], [4], [1], [12].

The theory of linear differential equations with deviating argument is well elaborated. The background for linear functional-differential equations can be found in the well known monographs: [1], [9].

(C) 2013 Mathematical Institute, Slovak Academy of Sciences. 2010 Mathematics Subject Classification: $34 K 06,34 \mathrm{~K} 19,34 \mathrm{~K} 25$.

Keywords: linear functional-differential equations, factorization, integral manifold of solutions. 
Consider the linear system of equations with a complex deviating

$$
\frac{d X(t)}{d t}=A X(t)+\mu \sum_{k=1}^{n} A_{k} X\left(t+\tau_{k}\right)
$$

where $\mu$ is a small parameter, $\tau_{k},(k=1, \ldots n)$ are complex constants, and $A, A_{k}$, are nonsingular constant matrices, $\operatorname{dim} X(t)=m . A, A_{k}, X(t)$ are supposed to be complex.

We seek a solution of system (1) in the form

$$
X(t)=e^{p t} K, \quad K=\text { const }
$$

For the complex number $p$ we obtain the equation

$$
\operatorname{det}\left(p I-A-\mu \sum_{k=1}^{n} A_{k} e^{p \tau_{k}}\right)=0 .
$$

In what follows we search for the factorization

$$
p I-A-\mu \sum_{k=1}^{n} A_{k} e^{p \tau_{k}}=(p I-C(\mu))(I+\mu G(p, \mu)),
$$

where $G(p, \mu)$ is a matrix with integer elements. Equation (2) could be written in the form

$$
C(\mu)-A-\mu \sum_{k=1}^{n} A_{k} e^{p \tau_{k}}=p \mu G(p, \mu)-\mu C(\mu) G(p, \mu) .
$$

By setting $\mu B(\mu)=C(\mu)-A$ we get the matrix equation

$$
B(\mu)-p G(p, \mu)=F(p, \mu)
$$

where

$$
F(p, \mu) \equiv \sum_{k=1}^{n} A_{k} e^{p \tau_{k}}-(A+\mu B(\mu)) G(p, \mu)
$$

The matrix equation (3) defines the matrices $G(p, \mu), B(\mu)$ and it may be solved by the method of successive approximations. A direct application of the contraction mapping theorem to the equations (3) seems not possible.

It is shown that for a sufficient small values of $|\mu|>0$ there is a system without deviating

$$
\frac{d X(t)}{d t}=(A+\mu B(\mu)) X(t)
$$

whose solutions are solutions to system (11). 


\section{FACTORIZATION OF LINEAR DIFFERENTIAL-DIFFERENCE EQUATIONS}

\section{Successive approximations}

The matrix equation (3) can be solved by the method of successive approximations.

We start this process by setting $G_{0}(p, \mu)=0, B_{0}(\mu)=0$ and

$$
\begin{gathered}
B_{j+1}(\mu)-p G_{j+1}(p, \mu)=F_{j}(p, \mu), \\
F_{j}(p, \mu) \equiv \sum_{k=1}^{n} A_{k} e^{p \tau_{k}}-\left(A+\mu B_{j}(\mu)\right) G_{j}(p, \mu), \quad \text { for } j=0,1,2, \ldots
\end{gathered}
$$

From this we obtain the matrices

$$
\begin{gathered}
B_{j+1}(\mu)=F_{j}(o, \mu)=\frac{1}{2 \pi} \int_{0}^{2 \pi} F_{j}\left(r e^{i \varphi}, \mu\right) d \varphi, \\
G_{j+1}(p, \mu)=\frac{1}{p}\left(B_{j+1}(\mu)-F_{j}(p, \mu)\right) .
\end{gathered}
$$

For $|p|=r$ it follows that

$$
\begin{gathered}
\left\|B_{j+1}(\mu) \mid \leq \max _{0 \leq \varphi \leq 2 \pi}\right\| F_{j}\left(r e^{i \varphi}, \mu\right) \|, \\
\max _{|p|=r}\left\|G_{j+1}(p, \mu)\right\| \leq \frac{2}{r}\left\|F_{j}\left(r e^{i \varphi}, \mu\right)\right\| .
\end{gathered}
$$

Now we have to establish the boundedness for the sequences

Let us denote

$$
\left\{\left\|B_{j}(\mu)\right\|\right\}, \quad\left\{\max _{|p|=r}\left\|G_{j}(p, \mu)\right\|\right\} .
$$

$$
\tau=\max _{|p|=r} \sum_{k=1}^{n}\left\|A_{k}\right\| e^{p \tau_{k}}=\sum_{k=1}^{n}\left\|A_{k}\right\| e^{r\left|\tau_{k}\right|}, \quad \tau=\tau(r) .
$$

Then from the estimate (17) we get

$$
\left\|B_{j+1}(\mu)\right\| \leq \tau+\left(\|A\|+|\mu|\left\|B_{j}(\mu)\right\|\right) \frac{2}{r}\left\|B_{j}(\mu)\right\| .
$$

Theorem 1. If the inequality

$$
\frac{2}{r}-\sqrt{2 r|\mu| \tau} \geq\|A\|
$$

is satisfied, then the sequences

$$
\left\{\left\|B_{j}(\mu)\right\|\right\}, \quad\left\{\max _{|p|=r}\left\|G_{j}(p, \mu)\right\|\right\}
$$

are bounded. 
Proof. The sequence of matrices $B_{j}(\mu)(\mathrm{j}=0,1,2, \ldots)$ is bounded in norm, if the equation in $z$

$$
z=\tau+\|A\| \frac{2}{r} z+\frac{2}{r}|\mu| z^{2}
$$

has two positive roots. To this end it suffices to satisfy the inequality

$$
1-\frac{2}{r}\|A\|>2 \sqrt{\frac{r}{2}|\mu| \tau,}
$$

which coincides with the condition (9).

For any given matrix $\mathrm{A}$ we can find $r>2\|A\|$ and a value $\mu_{0}$ such that for $|\mu| \leq \mu_{0}$ the inequality (9) is fulfilled.

For $z=\max _{j}\left\|B_{j}(\mu)\right\|$, from (10) we obtain

$$
z=\sqrt{\frac{r \tau}{2|\mu|}}, \quad\left\|B_{j}(\mu)\right\| \leq \sqrt{\frac{r \tau}{2|\mu|}} .
$$

Under the condition (9), from the inequality (77), it follows that the sequence $\max _{|r|=r}\left\|G_{j+1}(p, \mu)\right\|,(\mathrm{n}=0,1,2, \ldots)$ is bounded

$$
\max _{|r|=r}\left\|G_{j+1}(p, \mu)\right\| \leq \frac{2}{r} \sqrt{\frac{r \tau}{2|\mu|}}=\sqrt{\frac{2 \tau}{r|\mu|}},
$$

where $\tau$ is given by (8).

\section{Convergence of successive approximations}

We prove of convergence of the matrix sequences $\left\{B_{j}(\mu)\right\}$ and $\left\{G_{j}(p, \mu)\right\}$.

Theorem 2. If the inequality

$$
\frac{2}{r}-\sqrt{2 r|\mu| \tau}>\|A\|
$$

is satisfied, then the sequences $\left\{B_{j}(\mu)\right\}$ and $\left\{G_{j}(p, \mu)\right\}(j=0,1,2, \ldots)$ converge when $|p|=r$.

Pr o of. From (6) we have the equalities

$$
\begin{aligned}
B_{j+1}(\mu)-B_{j}(\mu) & =\frac{1}{2 \pi} \int_{0}^{2 \pi}\left(F_{j}\left(r e^{i \varphi}, \mu\right)-F_{j-1}\left(r e^{i \varphi}, \mu\right)\right) d \varphi, \\
G_{j+1}(p, \mu)-G_{j}(p, \mu) & =\frac{1}{p}\left(B_{j+1}(\mu)-B_{j}(\mu)-F_{j}(p, \mu)+F_{j-1}(p, \mu)\right) .
\end{aligned}
$$




\section{FACTORIZATION OF LINEAR DIFFERENTIAL-DIFFERENCE EQUATIONS}

Let us estimate the difference

$$
\begin{aligned}
F_{j}(p, \mu)-F_{j-1}(p, \mu)= & -A\left(G_{j}(p, \mu)-G_{j-1}(p, \mu)\right) \\
& -\mu B_{j}(\mu)\left(G_{j}(p, \mu)-G_{j-1}(p, \mu)\right) \\
& -\mu\left(B_{j}(\mu)-B_{j-1}(\mu)\right) G_{j-1}(p, \mu) .
\end{aligned}
$$

For $|p|=r$ we obtain the inequalities

$$
\begin{aligned}
\left\|B_{j+1}(\mu)-B_{j}(\mu)\right\| \leq & \sqrt{\frac{2|\mu| \tau}{r}}\left\|B_{j}(\mu)-B_{j-1}(\mu)\right\| \\
& +\left(\|A\|+\sqrt{\frac{1}{2} r|\mu| \tau}\right)\left\|G_{j}(p, \mu)-G_{j-1}(p, \mu)\right\|, \\
\left\|G_{j+1}(p, \mu)-G_{j}(p, \mu)\right\| \leq & \frac{1}{r}\left(1+\sqrt{\frac{2}{r}|\mu| \tau}\right)\left\|B_{j}(\mu)-B_{j-1}(\mu)\right\| \\
& +\frac{1}{r}\left(\|A\|+\sqrt{\frac{1}{2} r|\mu| \tau}\right)\left\|G_{j}(p, \mu)-G_{j-1}(p, \mu)\right\| .
\end{aligned}
$$

We introduce the notation

$$
\alpha=\sqrt{\frac{2}{r}|\mu| \tau}, \quad \beta=\|A\|+\sqrt{\frac{1}{2} r|\mu| \tau},
$$

in order to get simple sufficient conditions for convergence of the successive approximations (6). This requires that the eigenvalues of the matrix

$$
R=\left(\begin{array}{cc}
\alpha & \beta \\
r^{-1}(1+\alpha) & r^{-1} \beta
\end{array}\right)
$$

are less than 1 in modulus. And this leads to the inequality $\alpha+\frac{2 \beta}{r}<1$, which is the same as (13).

\section{The main result}

Summarizing, we have

Theorem 3. If the inequality

$$
\frac{2}{r}-\sqrt{2 r|\mu| \tau}>\|A\|, \quad \text { where } \tau=\sum_{k=1}^{n}\left\|A_{k}\right\| e^{r\left|\tau_{k}\right|}, \quad r=|p|,
$$

is satisfied, then the system (10)

$$
\frac{d X(t)}{d t}=A X(t)+\mu \sum_{k=1}^{n} A_{k} X\left(t+\tau_{k}\right)
$$


with complex deviating argument has an integral manifold of solutions given by the system

$$
\frac{d X(t)}{d t}=C(\mu) X(t), \quad \text { where } \quad C(\mu)=(A+\mu B(\mu)) .
$$

Remark 1. Under the assumptions of Theorem 3 and provided that the real numbers $\tau_{k}<0$ the stability of solutions of the system (11) is equivalent to the stability of solutions of the system (14) (13]).

The invariant subspace of the perturbed system was explicitly constructed using successive approximations in [6], 13].

Remark 2. The unstable subspace of linear ordinary differential equations persists under small linear perturbations - even in the presence of delays, this has been proved in [5], [7].

By this way one could investigate asymptotic properties of solutions of differential-delay equations.

\section{REFERENCES}

[1] AZBELEV, N. V.-MAKSIMOV, V. P.-RAKHMATULLiNA, L. F.: Methods of Modern Theory of Linear Functional Differential Equations. R\&C Dynamics, Moscow-Izhewsk, 2000. (In Russian)

[2] BERKOVICH, L.: Method of factorization of ordinary differential operators and some of its applications, Appl. Anal. Discrete Math. 1 (2007), 122-149.

[3] DIBLÍK, J.: Asymptotic representation of solutions of equation $\dot{y}(t)=\beta(t)[y(t)-$ $-y(t-\tau(t))]$, J. Math. Anal. Appl. 217 (1998), 200-215.

[4] ČERMÁK, J.: On the differential equation with power coefficients and proportional delays, Tatra Mt. Math. Publ. 38 (2007), 57-69.

[5] JANGLAJEW, K.: Construction of an integral manifold for a linear delay system, in: Difference Equations, Special Functions and Orthogonal Polynomials, Proc. of the Internat. Conf. (S. Elaydi et al., eds.), Munich, 2005, World Scientific Publ., Hackensack, NJ, 2007, pp. 286-293.

[6] JANGLAJEW, K.: On the reduction principle of difference equations, Dyn. Contin. Discrete Impulsive Syst. 6 (1999), 381-388.

[7] JANGLAJEW, K.-VALEEV, K.: Construction of an integral manifold for linear differential-difference equations, Opuscula Math. 26 (2006), 465-470.

[8] JANGLAJEW, K.-VALEEV, K.: The factorization of the difference operator, Comput. Math. Appl. 42 (2001), 729-733.

[9] KOLMANOVSKII, V.-MYSHKIS, A.: Introduction to the Theory and Applications of Functional-Differential Equations, in: Math. Appl. (East European Ser.), Vol. 463, Kluwer Acad. Publ., Dordrecht, 1999.

[10] LITTLEJOHN, L. L.-LÓPEZ, J. L.: Variation of parameters and solutions of composite products of linear differential equations, J. Math. Anal. Appl. 369 (2010), 658-670.

[11] MYSHKIS, A. D.: On certain problems in the theory of differential equations with deviating argument, Uspekhi Mat. Nauk 32 (1977), No. 2, 173-202. (In Russian) 


\section{FACTORIZATION OF LINEAR DIFFERENTIAL-DIFFERENCE EQUATIONS}

[12] RYABOV, YU. A.: Certain asymptotic properties of linear systems with small time delay, Sov. Math. Dokl. 4 (1963), 928-930.

[13] VALEEV, K. G.: Splitting of Matrix Spectra. Vishcha Shkola, Kiev, 1986. (In Russian)

[14] VALEEV, K.-JANGLAJEW, K.: The factorization of the differential expression, SVMO Preprint Ser., Vol. 53, Srednevolgskoye Matematicheskoye Obshchestvo, Saransk, 2003.

Received July 16, 2012

Klara R. Janglajew

Institute of Mathematics

University of Biatystok

Akademicka 2

15-267 Biatystok

POLAND

E-mail: jang@math.uwb.edu.pl

Kim G. Valeev

Department of Mathematics

Kyiv National Economic University 03049 Kyiv

Kurskaya 3/13

UKRAINE

E-mail: kimvaleev36@mail.ru 